\title{
Las eRúbricas ante la evaluación de competencias transversales en Educación Superior ${ }^{1}$
}

\section{Using eRúbricas for assessing transversal competences in Higher Education}

\author{
Itziar REKALDE RODRÍGUEZ y Karmele BUJÁN VIDALES \\ Universidad del País Vasco
}

Recibido: Marzo 2013

Aceptado: Julio 2013

\begin{abstract}
Resumen
Este trabajo se ha desarrollado en la Universidad del País Vasco-Euskal Herriko Unibertsitatea y en dos contextos de investigación diferentes como son la Facultad de Filosofía y Ciencias de la Educación, y la Escuela de Magisterio de Donostia, así como para dos grados distintos: Educación Primaria y Educación Social. La estructura modular que articula estos grados y el compromiso por el desarrollo de dos de las competencias transversales que comparten (trabajo en equipo y comunicación oral), nos ha facilitado el trabajo colaborativo por parte de las docentes en la construcción de un diseño compartido de las tareas de aprendizaje e instrumentos de evaluación, eRúbrica, así como los criterios que en ella se recogen. La eRúbrica ha favorecido la construcción de un entorno virtual compartido por el profesorado y alumnado, donde se ha experimentado al objeto de llevar a cabo un seguimiento del desarrollo y evaluación de dichas competencias. El trabajo pone en valor, por una parte, la utilización de las eRúbricas en el seguimiento y evaluación de competencias transversales $\mathrm{y}$, por otra, el trabajo colaborativo desplegado por las docentes como un elemento clave y de éxito en el mismo.
\end{abstract}

Palabras clave: Competencias; Trabajo en equipo; Comunicación oral; Evaluación; Universidad.

\begin{abstract}
This work was carried out at University of the Basque Country in two different research contexts - the School of Philosophy and Education Science and the Teachers College in Donostia-and two different degree programs: Primary Education and Social Education. The modular structure of the two degree programs and the fact that they are both committed to the development of the transversal competences of teamwork and oral communication has allowed

\footnotetext{
${ }^{1}$ Este trabajo ha sido desarrollado en el marco del proyecto de investigación titulado Servicio federado de eRúbrica para la evaluación de aprendizajes universitarios. Proyecto del Plan Nacional I+D+i 2010-2013. EDU2010-15432. Resolución de 30 de diciembre de 2009 (BOE de 31 de diciembre).
} 
instructors to collaborate on eRúbrica, a common architecture for learning tasks and assessment tools, and on the criteria included within the architecture. eRúbrica favors the construction of a virtual environment that is shared by instructors and students, where the objective is to track the development and assessment of the two transversal competences. This project highlights the usability of eRúbrica in tracking and assessing the development of transversal competences as well as the fact that the collaborative work carried out by instructors is a basic element that is key to its success.

Keywords: Competences; Teamwork; Oral communication; Assessment; University.

Desde que los organismos europeos generaron el concepto de alfabetización funcional (Unesco, 1970), entendiéndolo como la adquisición de aptitudes profesionales y de conocimientos utilizables en un medio determinado, se ha puesto de manifiesto la necesidad de replantearse los niveles suficientes de capacitación que posibilite a los sujetos una socialización adecuada en un contexto de cambios constantes y acelerados. La evolución de dicha reflexión ha generado en las últimas décadas el surgimiento del concepto de competencia, y el establecimiento de una serie de Key skills (competencias clave) (Gimeno, 2008; Monereo, 2009), que se están convirtiendo paulatinamente en el eje orientador de la práctica educativa en los diversos niveles de enseñanza y de las políticas generales de educación de los Estados y Regiones miembros de la Unión Europea (Informe Delors, 1996; Proyecto DeSeCo de la OCDE, 1997; Eurydice, 2002; LOE, 2006). Nos referimos a competencias como por ejemplo; aprender a aprender, autonomía e iniciativa personal, competencia lingüística, competencia matemática... que se desarrollan en los niveles obligatorios de la enseñanza. En líneas generales, y teniendo en cuenta el entorno complejo y cambiante en el que nos movemos, la radiografía social que se precisa debe ahondar más que nunca en un modelo de formación basado en el respeto a la diversidad, en la educación en valores y, en el desarrollo de competencias a lo largo de la vida, Lifelong learning.

Dentro de este entramado y respecto a las llamadas competencias transversales, fueron Prahalad y Hamel (1990) quienes defendieron y trataron de demostrar que un número reducido de competencias Core competencies eran la explicación del éxito de las organizaciones. Estos trabajos de management y gestión empresarial tuvieron, en paralelo, un análisis y aplicación en el ámbito de la enseñanza. Para comprender y funcionar bien en este mundo, los individuos necesitan, por ejemplo, dominar las tecnologías cambiantes y comprender enormes cantidades de información disponible. De la misma manera que enfrentar desafíos colectivos como sociedades, -tales como el balance entre el crecimiento económico y la sostenibilidad ambiental, y la prosperidad con la equidad social-. En estos contextos, las competencias que los individuos necesitan satisfacer para alcanzar sus metas se han ido haciendo más complejas, requiriendo de un mayor dominio de ciertas destrezas definidas estrechamente. Tal y como sostienen los Ministerios de Educación de la OCDE (Salganik, Rychen, Moser y Konstant, 1999; Rychen y Salganik, 2004) al afirmar que el desarrollo sostenible y la cohesión social dependen críticamente de las competencias de toda nuestra población, 
con competencias que se entiende cubren el conocimiento, las destrezas, las actitudes y los valores.

Las competencias clave constituyen los saberes elementales escolarizables que, entendemos, capacitan a los sujetos para enfrentarse a los nuevos retos del presente y del futuro. Suponen la integración de conocimientos, habilidades, actitudes y valores (Perrenoud, 2004) que una persona pone en juego en una situación concreta demostrando que es capaz de dar respuesta. Constituyen, por tanto, estas competencias la piedra angular sobre la que construir los nuevos procesos formativos para que se haga posible la transformación tan reclamada en esta sociedad del conocimiento y de cambio globalizado (Hargreaves, 2003). Es más, esta formación no debe entenderse como un aprendizaje fragmentado, tal como se entendían las competencias desde un enfoque conductista, sino como un aprendizaje integrador, donde un sujeto competente será aquel que sepa leer el entorno (los contextos) y los múltiples discursos que circulan, para tomar una posición frente a ellos y actuar (Jurado, 2009).

Desde este mismo enfoque, las competencias genéricas o transversales, tanto instrumentales, interpersonales como sistémicas (Tuning Proyect, 2000), son priorizadas en la formación superior, complementándose con las competencias específicas de cada perfil profesional con el propósito de capacitar a la persona en la comprensión para la acción en el desarrollo de su profesión (Pérez Gómez, 2008).

Este nuevo discurso le supone al sistema educativo superior plantearse un atractivo como difícil reto: transitar de un modelo de docencia centrado en la enseñanza, a otro centrado en los procesos de aprendizaje, donde el alumnado sea capaz de plantearse preguntas, investigar, seleccionar y organizar información, analizar datos, extraer conclusiones, expresarse adecuadamente (Blanco, 2009). El estudiante debe promover disposición a implicarse de manera responsable en el ejercicio de su profesión y también como miembro de una ciudadanía activa (Martínez y Payà, 2007). Estamos, por tanto, refiriéndonos a un importante cambio formal y organizativo, pero sobre todo a un cambio de cultura educativa (Rekalde et al., 2012). Es por ello que este nuevo planteamiento supone un cuestionamiento no sólo de los contenidos sobre los que se debe formar al alumnado, sino también sobre el propio proceso de enseñanzaaprendizaje, en el que se debe dar lugar al desarrollo de nuevas propuestas metodológicas, donde se dé paso a formas interdisciplinares de aprendizaje en los que el protagonista activo sea el alumnado (Imbernon y Medina, 2006; Moya, 2008). De ahí que los métodos de enseñanza-aprendizaje deben tener un enfoque globalizador, donde se utilicen metodologías variadas con secuencias didácticas enfocadas bajo el método de aprendizaje basado en problemas, proyectos, análisis de casos, investigaciones del medio, aprendizaje y servicio solidario... (Zabala y Arnau, 2007). Entendemos que optar por una educación en competencias representa la búsqueda de estrategias de enseñanza que sitúan su objeto de estudio en la forma de dar respuesta satisfactoria a situaciones reales y, por lo tanto, complejas. Dado que estas situaciones reales nunca serán aquellas en las que se va a encontrar el alumnado en la realidad, se entiende que se desarrollan los esquemas de actuación y, su selección y puesta en práctica, en distintos contextos generalizables. Estas tareas deben integrar conocimientos, destrezas y actitudes, y estar dotadas de un carácter de autenticidad, es 
decir; deben ser relevantes, reflejar la complejidad de la realidad, y anclar con tareas de mayor envergadura.

Esta nueva mirada, como venimos subrayando, posa su atención en las actividades de aprendizaje; en generar espacios de gestión del conocimiento, de las emociones, actitudes... que conforman el universo del desarrollo competencial. Estamos hablando, por tanto, de propiciar al alumnado verdaderos contextos de enriquecimiento académico, profesional y personal, donde las tareas propuestas sean: a) auténticas; que sitúen al alumnado ante problemas, casos... situaciones, en definitiva, lo más reales posibles, que le hagan ponerse con facilidad en el rol profesional y sentirse como tales, para enfrentarse a ellas de manera adecuada y significativa (Biggs, 1999); b) integradas; que estén entroncadas en el proyecto curricular del grado, donde el espacio, tiempo y agrupamiento sean variables dependientes del proyecto y no obstáculos para su desarrollo, y c) activas; que integren conocimientos y procedimientos dejando cierto margen de indeterminación para que logren resolver la tarea con márgenes de creatividad y trabajo en equipo, como en la vida real de los profesionales (Rekalde, 2011). Resumiendo, el punto crítico de los procesos de desarrollo de competencias en educación superior, radica en la selección de situaciones altamente relevantes para el quehacer profesional, lo que implica generar espacios de sinergia entre el curriculum y la práctica profesional.

Este cambio en la arquitectura de los procesos de enseñanza-aprendizaje nos lleva, también, a posar la mirada en la evaluación de los resultados de aprendizaje y la calificación de los productos derivados de las tareas auténticas (Gulikers et al., 2006; Blanco, 2008). Por lo que conlleva todo un cambio en los elementos intrínsecos del diseño curricular $\mathrm{y}$, especialmente en el planteamiento de la evaluación por competencias. De ahí que Boud (1995) nos recuerde que los estudiantes pueden, con dificultad, escapar de los efectos de una mala enseñanza, pero no (por definición, si quieren graduarse) de los efectos de una mala evaluación. La evaluación, sigue siendo uno de los elementos curriculares que más problemas acarrea tanto al docente como al alumnado, y es en este nuevo marco de construcción de proyectos educativos articulados a través de competencias, donde se nos presenta clave el tema de la evaluación si queremos plantear nuestros diseños formativos con rigor, coherencia y en sintonía.

Uno de los instrumentos que garantiza la evaluación auténtica y alternativa es la rúbrica. Las rúbricas son guías o escalas de evaluación donde se establecen niveles progresivos de dominio o pericia relativos al desempeño que una persona muestra respecto de un proceso o producción determinada (Stevens y Levi, 2005). Es a través de las rúbricas como conseguimos elementos que guíen y propicien un aprendizaje profundo (Biggs, 1999; Goñi, 2005), y autónomo del alumnado (Buján, Rekalde, y Aramendi, 2011; Panadero, Alonso, y Huerta, 2012), dado que facilitan tanto la evaluación formativa (Panadero y Jonsson, 2013) como la evaluación de los resultados de aprendizaje y la calificación de los productos derivados de las tareas auténticas sujetas a criterios. Sirven, en definitiva, para evaluar pero, también, para apoyar los procesos formativos, donde los estudiantes adquieren un rol activo y de autorregulación. 
En nuestro caso, las rúbricas utilizadas han sido digitales, de ahí que nos refiramos a ellas como eRúbricas. La eRúbrica es una herramienta tecnológica y una metodología de evaluación formativa basada en competencias, que ayuda en la comunicación evaluativa entre docentes y estudiantes, y facilita la gestión de la autoevaluación de los aprendizajes por los estudiantes (Serrano y Cebrián, 2011), además de promover el diálogo, la reflexión y la socialización.

\section{Objetivos}

Analizar las fortalezas y las debilidades que puede presentar la utilización de eRúbricas en contextos de aprendizaje presenciales para el seguimiento y evaluación de las competencias transversales de trabajo en equipo y comunicación oral.

\section{Cuestiones metodológicas}

\section{El contexto. Pasos hacia un curriculum integrado}

La teoría curricular ha sufrido una gran transformación. Hasta no hace mucho tiempo la teoría curricular predominante utilizaba un esquema determinista de corte mecánico, en el que se suponía un hacer y entender la educación de manera lineal. Convertía, por tanto, el curriculum en un mecanismo rígido y cerrado, construido de manera artificiosa de espaldas al mundo real; al de la emergencia y las sorpresas. Hoy en día, el curriculum se concibe siguiendo un planteamiento sistémico, donde el curriculum es un sistema vivo, complejo, no lineal y caracterizado por la interdependencia y el feed-back entre sus elementos, la visión global y el entronque ecológico. Es decir, el curriculum como un instrumento abierto a la sociedad, al intercambio y adaptación, a la autorregulación, reflexión y evaluación fruto de la interacción (Doll, 1993). Esta concepción plural está dando pasos hacia una construcción de curriculas integrados, que giran en torno a la preocupación por la integración de los aprendizajes: dar sentido a éstos, en particular, precisando los tipos de situaciones en las cuales el alumnado va a movilizar los conocimientos adquiridos.

El concepto de currículo integrador va, por consiguiente, más allá de la única dimensión de integración de las disciplinas (interdisciplinario), tomada en cuenta por el enfoque anglosajón del concepto de integrative curriculum, pero incluye todas las dimensiones según las cuales el alumnado puede tejer una diversidad de lazos con sus conocimientos, para permitir una movilización efectiva de ellos: integración situacional, integración teoría-práctica, integración didáctica, interdisciplinaridad, transdisciplinaridad. Como nos recuerda, Roegiers (2007), el currículo integrador no existe en lo absoluto, pero traduce esencialmente un grado de preocupación por la integración. Zabalza (2012) diferencia seis estrategias de integración curricular: 1) la identificación de los semestres con bloques formativos; 2) el establecimiento de espacios comunes a varias disciplinas; 3) la fusión total o parcial de materias; 4) los clusters de materias impartidas por clusters de profesores y, 5) la organización curricular por módulos temáticos. Es esta última modalidad la que nos lleva a los 
modelos de integración más propios de los enfoques por competencias, y se corresponden a los niveles 9 de multidisciplinariedad, 10 de interdisciplinariedad y 11 de transdisciplinariedad de la escalera de Harden citada por Zabalza (2012). Las materias pasan a un papel secundario y su función es aportar aquellos conocimientos parciales que desde su campo de especialización pueden ayudar a resolver un problema planteado.

El contexto propiamente de esta investigación se sitúa en el marco del Grado de Educación Social y en el de Educación Primaria, donde la preocupación mencionada por la integración curricular ha estado presente. De hecho ambos currícula se articulan a través de módulos organizadores de las disciplinas de tal modo que dan sentido y significado a las mismas a lo largo de un cuatrimestre. El acercamiento mayor a la integración curricular se realiza con la Actividad Interdisciplinar de Módulo (AIM), donde el equipo docente diseña y pone en marcha, a partir de situaciones y problemas reales o cercanos a ella, la adquisición y desarrollo de las competencias correspondientes a ese nivel curricular (Rekalde, Martínez, y Marko, 2012).

A continuación ofrecemos una breve descripción de los dos contextos donde se ha desarrollado la investigación (tabla 1). La cursiva en la columna de las disciplinas significa la asignatura donde se da mayor concreción al contexto de la indagación:

\begin{tabular}{cccc} 
Grado Curso & Módulo & Disciplinas & $\begin{array}{c}\text { Actividad } \\
\text { Interdisciplinar de } \\
\text { módulo }\end{array}$ \\
\hline
\end{tabular}

$\begin{array}{lll} & & \text { 1. Investigación socioeducativa } \\ & \text { IV Módulo: } & \text { 2. Planificación de la intervención } \\ & \text { Planificación, Evaluación de programas y } & \text { servicios socioeducativos } \\ \text { Educación } & \text { gestión y } & \text { 4. Propuestas metodológicas } \\ \text { Social } & \text { evaluación de } & \text { generales de intervención y en } \\ & \text { programas } & \text { situaciones de exclusión } \\ & \text { socioeducativos } & \text { 5. Tecnologías de la información y } \\ & & \text { la comunicación en educación } \\ & & \text { social }\end{array}$

Diseño y defensa de un proyecto de intervención socioeducativa
1.- Bases de la escuela inclusiva

2.- Dificultades en el desarrollo y

IV Módulo: $L a$ diversidad en la escuela en el aprendizaje

3.- Bases didácticas del lenguaje en educación primaria en contextos multilingües

4.- Organización escolar
Elaboración de un artículo académico respecto a un caso: alumnado con dificultades en la escuela 
Podemos destacar que las dos AIM tienen características comunes en tanto que: a) representan un reto abordable para el alumnado; b) provocan un conflicto cognitivo y promueven la actividad mental necesaria para que se establezcan relaciones entre los nuevos contenidos y las competencias previas; c) fomentan una actitud favorable, porque son actividades motivadoras; d) conforman situaciones significativas y funcionales como profesional, y e) ayudan a desarrollar competencias específicas de la profesión y transversales tales como la comunicación oral y escrita, el trabajo en equipo o las habilidades relacionadas con el aprender a aprender, que le permitan ser cada vez más autónomo en sus aprendizajes.

Son concretamente en la adquisición y desarrollo de algunas de las competencias transversales de trabajo en equipo y comunicación oral donde hemos puesto nuestra atención.

\section{Los participantes}

Las disciplinas en las que hemos utilizado eRúbricas son Organización escolar en un grupo de 40 estudiantes donde se han formado 8 equipos de trabajo, y Propuestas metodológicas generales de intervención y en situaciones de exclusión en un grupo de 51 estudiantes, formándose 10 equipos. En ambas asignaturas ha participado el alumnado que asistía con regularidad a las sesiones presenciales y que, por tanto, se ha comprometido con una evaluación continua en su proceso de aprendizaje.

Respecto al perfil del alumnado el $73 \%$ han sido participantes femeninas, un $89 \%$ su edad está comprendida en el intervalo de 19-21 años y un 18\% compagina los estudios con un trabajo remunerado. Todos, además, han cursado las disciplinas en Euskara.

\section{El diseño de investigación}

El diseño de la investigación es el resultado de haber puesto en dialéctica instrumentos de corte cuantitativo, las eRúbricas, con estrategias de generación de datos de tipo cualitativo, las narraciones. Como señala LeCompte (1995), se han de reconciliar ambas perspectivas para conformar una conveniente compatibilidad y lograr una visión holística de la realidad. En nuestro trabajo, tanto unos instrumentos como otros nos han ayudado a describir, analizar e interpretar el uso de las eRúbricas para el seguimiento y desarrollo de las dos competencias transversales mencionadas (figura 1). 


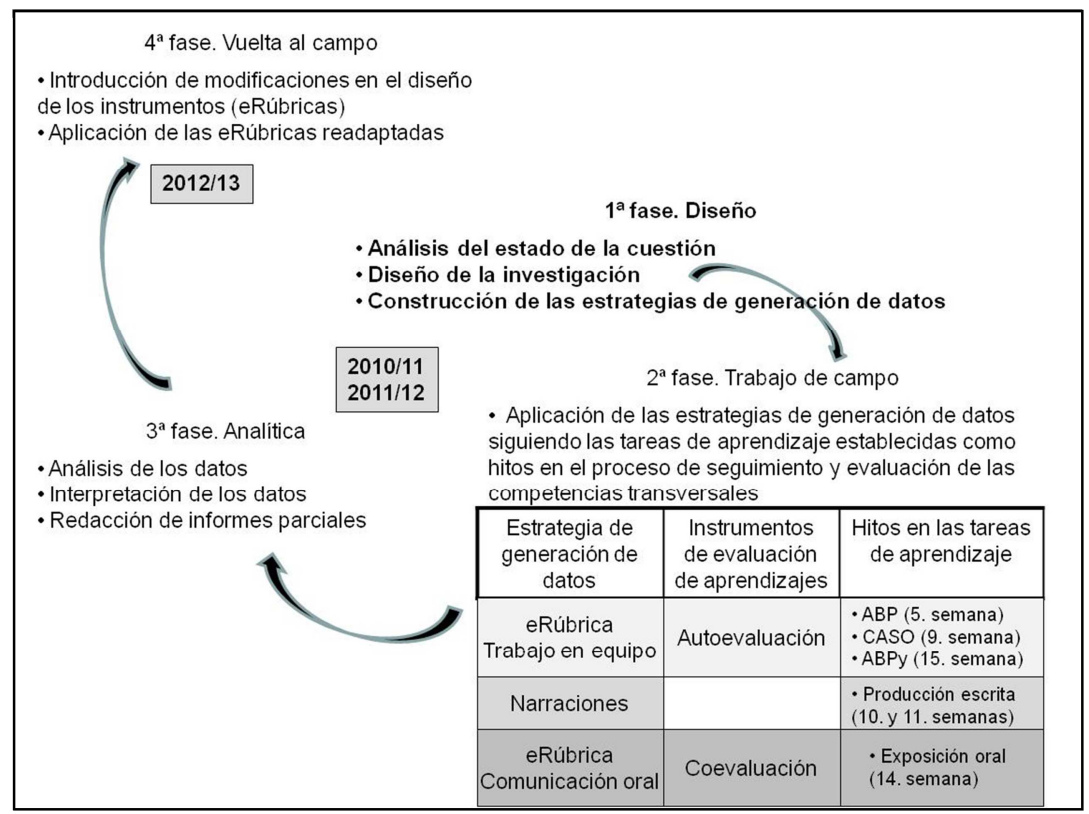

Figura 1. El diseño de la investigación

El instrumento objeto de indagación y, a la vez, generador de datos cuantitativos ha sido la eRúbrica. Si bien este instrumento tiene un fuerte componente descriptivo, éste se ha transformado en valor numérico al objeto de facilitar el uso digital de la herramienta por parte del alumnado.

En cuanto a las eRúbricas como estrategia para la recogida de información debemos puntualizar la diferencia entre la eRúbrica diseñada para el Trabajo en equipo y la Comunicación oral por que, como se expone a continuación, tienen características diferentes.

La competencia de Trabajo en equipo disfruta de las siguientes características:

a) instrumento para ser utilizado después de llevar a cabo una actividad o tarea didáctica significativa para el alumnado y con base en una metodología activa. Es decir, han hecho uso de la eRúbrica tras abordar un Problema (Aprendizaje Basado en Problemas -ABP-) ( $5^{\circ}$ semana), después de trabajar un caso (Método de Casos) (9 ${ }^{\circ}$ semana) y, al finalizar el diseño de un Proyecto (Aprendizaje Basado en Proyectos ABPy-) (15 semana). Si bien en las asignaturas se utilizan actividades prácticas diferentes, se escogieron estas tres a lo largo del cuatrimestre por constituir tres hitos en el proceso de aprendizaje que colocan al alumnado en una situación lo más real y cercana a su futuro desempeño profesional y, por la importancia del trabajo en equipo en su desarrollo. Por tanto, la competencia de Trabajo en equipo como cualquiera de las específicas, no se ha improvisado, sino que se han ofrecido situaciones al 
alumnado, preparadas previamente y cuidadas en sus detalles de procesos y de estructura, en las que desarrollar y adquirir dicha competencia (Rekalde y PérezSostoa, 2011).

b) instrumento que sirve de autoevaluación. Para tener éxito académico el alumnado necesita ser autónomo y ello se consigue si éste es capaz de dirigir, supervisar y corregir su modo de trabajar (Kitsantas y Zimmerman, 2009). La autoevaluación supone reflexionar y tomar conciencia de lo que se ha hecho permitiendo repetirlo, si ha sido favorable, y en caso contrario tomar conciencia de los errores y corregirlos. Como ya hemos señalado la herramienta diseñada para que el alumnado se autoevalúe en el desarrollo y adquisición de la competencia Trabajo en equipo corresponde al nivel de desempeño correspondiente a $2^{\circ}$ curso. Ello significa que en la trayectoria universitaria el alumnado lleva, aproximadamente, desarrollando esta competencia año y medio; es decir, que no comienza de cero.

Esta eRúbrica contempla las siguientes dimensiones: 1) fomento de la participación. Evalúa el tipo de participación y cómo se han sentido trabajando en equipo; 2) intervención en el clima del equipo. Evalúa el clima de trabajo y los sentimientos generados en el mismo; 3 ) asunción de roles. Evalúa el desempeño de roles tales como: secretario, portavoz, dinamizador, coordinador... y, 4) consecución de objetivos. La actitud del grupo frente a las tareas a desarrollar.

Respecto a la competencia de Comunicación oral, debemos concretar que se ha utilizado la eRúbrica como instrumento de coevaluación y después de que los diferentes equipos llevaran a cabo una exposición oral sobre un contenido curricular. Esta actividad ha tenido dos objetivos: por una parte, impulsar una tarea de indagación sobre un contenido curricular específico y, por otra, servir de entrenamiento para la exposición oral de la AIM ante un tribunal evaluador (14 semana). Por este motivo se han utilizado los mismos criterios establecidos para evaluar la competencia de comunicación oral por el tribunal, aunque con alguna especificidad y profundización en los mismos. Esta eRúbrica contempla las siguientes dimensiones: 1) nivel, estructuración, adaptación y cohesión de los contenidos; 2) actividad del equipo, organización y dinamización de la presentación; 3) comunicación oral y comunicación no verbal, y 4) utilización de los recursos de apoyo.

Cada equipo de trabajo disponía en la plataforma moodle de la asignatura el enlace directo que le llevaba a tener que identificarse en el servidor de la UPV/EHU y acceder así a la eRúbrica correspondiente del grupo que había hecho la exposición para poderles evaluar.

El instrumento cualitativo que se ha utilizado para recabar información han sido las narraciones. A este respecto, debemos puntualizar que el giro discursivo en el campo de las ciencias sociales ha abierto el camino a la aparición de nuevos paradigmas críticos alejados de los criterios clásicos de validación positivista. Las narrativas constituyen nuevos objetos de análisis, atribuyéndoles una importancia relevante en la capacidad de descripción de realidades subjetivas (Pujadas, 2004; Biglia, y BonetMartí, 2009). En nuestro caso, el alumnado disponía previamente de las dos preguntas sobre las que se deseaba indagar, que eran: desarrollo de estas competencias a lo largo 
de la escolaridad y utilidad de las eRúbricas para el seguimiento y desarrollo de dichas competencias de cara al desempeño profesional futuro. Las narraciones las han llevado a cabo todo el alumnado como una tarea más entroncada en el contenido de los programas de las asignaturas.

Las modalidades de triangulación que, siguiendo a Denzin (1978) e Janesick (1994), hemos utilizado han sido las siguientes: la triangulación de técnicas y de investigadores.

La investigación que presentamos se ha desarrollado a lo largo de dos cursos académicos (2010/11 y 2011/12), y contempla cuatro fases en su diseño: a) primera fase en la que se realizó el análisis del estado de la cuestión, y el diseño de los instrumentos, b) segunda fase en la que ha tenido cabida la implementación, y que se describe de manera pormenorizada en el texto y en la figura 1, c) tercera fase donde se ha procedido al análisis e interpretación de los datos generados, así como a la redacción de informes parciales y, d) la vuelta al campo con nuevas modificaciones sobre los instrumentos pero con otros grupos de alumnado (2012/13).

Los resultados de las eRúbricas se analizaron con los estadísticos básicos del programa de análisis de datos SPSS 17.0 (análisis univariados) y las reflexiones vertidas en las entrevistas a través del programa ANSWR. La cercanía a los informantes ha permitido las continuas idas y venidas al campo de trabajo.

\section{Resultados}

Comenzaremos por los resultados obtenidos en la competencia de Trabajo en equipo.

Se les ha ofrecido tres hitos a lo largo del cuatrimestre, tras los cuales autoevaluarse. Esta acción les ha servido para ir paulatinamente apropiándose del instrumento eRúbrica y tomar conciencia del valor de los criterios. Además, la autoevaluación, tenía después de la misma un feed-back; es decir, que no ha sido aleatoria sino pautada por el profesorado. Al hilo de lo anterior, se les ha facilitado las respuestas a sus autoevaluaciones de manera que pudieran ir viendo el avance en el desarrollo de la competencia. La gráfica 1 nos desvela datos que, a nuestro entender, son muy significativos. Iremos describiéndolos aplicando un análisis puntual cronológico de cada una de las tres tareas llamadas hitos de aprendizaje y, un análisis procesual destacando la progresión de las dimensiones a lo largo del tiempo.

Es la dimensión relativa a Objetivos aquella que independientemente del reto de aprendizaje (ABP, Caso, ABPy) obtiene valores más altos. Ésta se refiere a la actitud del grupo frente a las tareas, y recoge indicadores relativos a la rapidez con que el equipo empieza a trabajar; la finalización de las tareas; la presencia de los objetivos de la tarea a lo largo de la realización de la misma... No obstante, se ve una clara progresión entre la tarea realizada bajo el $\mathrm{ABP}$ y la última a través de ABPy.

La dimensión denominada Participación es claramente aquella en la que se experimenta un fuerte salto entre la segunda y tercera tarea o hito de aprendizaje. 
La dimensión de Roles no muestra variaciones significativas a lo largo del cuatrimestre, lo mismo que le ocurre a la relativa a Clima. La primera se refiere a la asunción y ejecución de las funciones de secretario, portavoz y coordinador del equipo (rotativo), cómo se han sentido en los diferentes papeles y si les gusta el desempeñar cada uno de los roles. Una de las voces nos señala que: "Nunca había sido consciente de los roles a desempeñar en un equipo, y el practicarlos me está sirviendo para asumir posiciones distintas frente a los demás. A tomar decisiones que eso antes me costaba mucho".

Por lo que respecta a la segunda, se intentan evaluar indicadores relativos a la gestión del tiempo, a la libertad dentro del equipo, a los compromisos que se asumen, la aportación individual a la comunicación y la interacción... Una voz comenta al respecto que: "Yo estoy aprendiendo la importancia que tiene el sentimiento de pertenencia al grupo, cada vez lo valoro más". No obstante, aunque no es destacable, pero sí que se registran puntuaciones algo más altas al finalizar el cuatrimestre para estas dos dimensiones.

Son, sin duda, las dimensiones de Participación y Objetivos las que a lo largo del proceso experimentan una mayor progresión, probablemente debido a la presión de la entrega del producto AIM que se corresponde con el ABPy.

Si realizamos un análisis asentándonos en las tareas de aprendizaje, nos damos cuenta que en las tres el patrón de las dimensiones a lo largo del cuatrimestre es el mismo; siendo la participación la dimensión menos valorada, después el clima, a continuación los roles y, por último, la más valorada los objetivos.

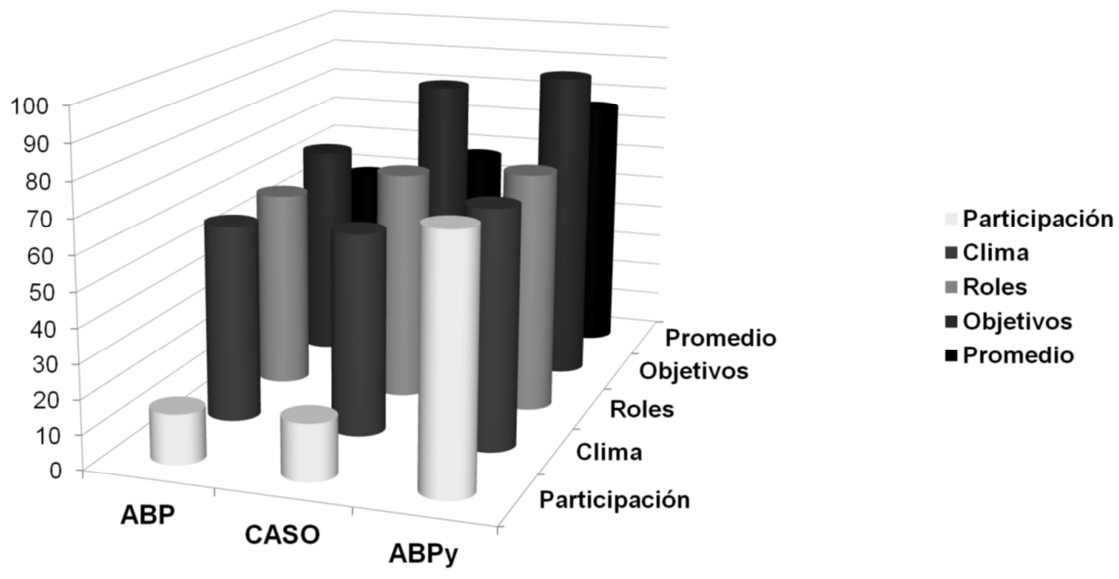

Grafico 1. Competencia Trabajo en equipo y sus dimensiones de análisis

El 60\% del alumnado afirma no haber trabajado en grupo antes de llegar a la universidad. Rescatamos algunas de las voces que afirman este hecho: "Lo de trabajar en equipo ha sido una cosa nueva para mí"; otro estudiante comenta que "Cuando he 
llegado a la universidad es cuando estoy aprendiendo a trabajar en equipo", y otro nos dice al respecto que "Ahora los trabajos importantes los hacemos en equipo".

En caso de haber tenido experiencias previas en trabajar en equipo, éstas han sido puntuales sin continuidad en el proceso educativo, y calificadas como: malas, frustrantes... competitivas y no cooperativas. Tal y como lo recogen los informantes: "Creo recordar que sólo en una asignatura de bachiller trabajé en grupo, no recuerdo más ocasiones" y otra de las voces aluden que "Antes de cursar educación social estuve en otra universidad donde también se trabajaba en grupo pero para conseguir que tu proyecto fuera mejor que el de los otros".

En cuanto a la competencia de Comunicación oral, se ha evaluado a través de una eRúbrica de coevaluación, donde cada equipo después de exponer un tema concreto vinculado a los contenidos curriculares del programa, el resto debía valorar su actuación. La evaluación era inmediata y sujeta a los mismos criterios que se iban a utilizar en la defensa ante tribunal de la AIM. El gráfico 2 nos muestra que en los 18 equipos (cada uno bajo un nombre) la dimensión que de manera constante ha sido puntuada con valores más bajos es la del uso apropiado de recursos de apoyo; recursos que hayan facilitado la presentación del contenido oral y el uso didáctico de los mismos.

En cambio, la dimensión relativa al contenido es la que ha sido puntuada con valores más altos, y se refiere a la estructura, adaptación y cohesión de los contenidos del discurso.

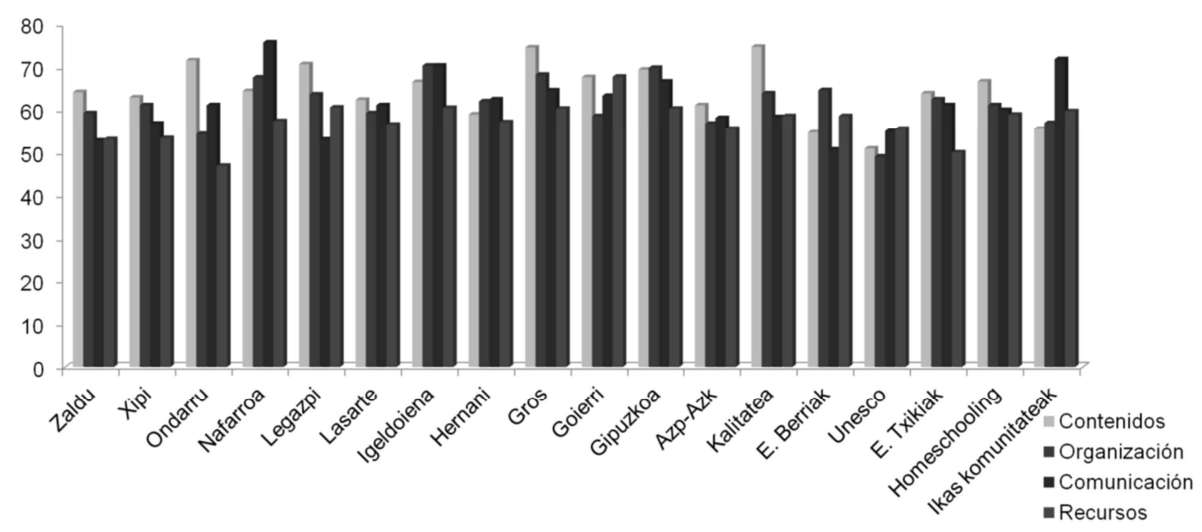

Grafico 2. Competencia Comunicación oral y sus dimensiones de análisis

Algunos pasajes de las narraciones del alumnado nos subrayan que: "A nosotras nos ha venido muy bien para preparar la defensa del proyecto ante el tribunal", o esta otra voz que dice: "Te hace ser consciente de los puntos débiles del grupo, y poner así más atención en ellos e intentar mejorarlos". 


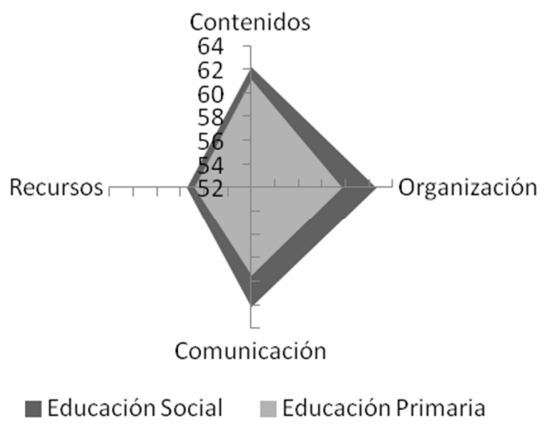

Gráfico 3. Competencia Comunicación oral. Comparativa entre el grado de Educación social y Educación Primaria

En cuanto a las diferencias que podemos apreciar entre los equipos de los dos grados, podemos señalar que las diferencias no son significativas respecto a las dimensiones de contenido y recursos que son, en ese orden, la mejor y la peor valorada respectivamente. Es decir, en ambos grados valoran con la puntuación más alta la dimensión denominada Contenidos, en la que se tiene en cuenta la estructuración, adaptación y cohesión de los contenidos a la hora de exponerlos delante del grupoclase. Esta misma coincidencia se vuelva a producir con la dimensión denominada Recursos pero en este caso a la baja, dado que en ambos grados las puntuaciones más bajas se recogen en esta dimensión relativa a la utilización de los recursos de apoyo para la exposición delante del grupo-clase. En cambio la diferencia sí que se amplía entre los dos grados en las dimensiones de Organización y Comunicación (Gráfico 3). En líneas generales, las puntuaciones de los grupos de Primaria hacia sus compañeros han sido más bajas, que en los equipos de Educación Social.

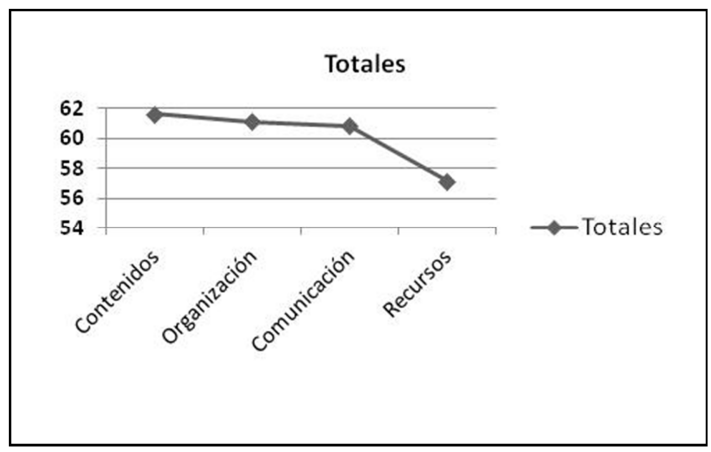

Gráfico 4. Valoración de las dimensiones de la competencia Comunicación oral en los dos grados 
Sin embargo, tanto en una titulación como en la otra la tendencia en las puntuaciones es la misma, siendo la dimensión más valorada la relativa al Contenido, después la Organización y dinámica de exposición, a continuación la Comunicación verbal y no verbal y, por último, la de los Recursos utilizados (Gráfico 4). Esto puede significar para los dos grados que la preparación del texto escrito donde se recoge el contenido teórico-práctico sobre el cual se va a articular el discurso oral, sigue siendo la dimensión mejor trabajada y cuidada por los equipos de trabajo frente a otros aspectos que también van a ser tenidos en cuenta en la evaluación. Entre estos últimos destacamos, por su puntuación más baja, la ayuda que los soportes técnicos pueden facilitar en el desarrollo de la exposición y que, sin embargo, se siguen descuidando.

\section{Discusión}

No podemos negar que el uso de rúbricas en los últimos años, a raíz de las nuevas orientaciones marcadas por el EEES, ha experimentado un aumento significativo en la educación superior. Las luces y las sombras que éstas presentan han sido ampliamente estudiadas. En este contexto, el universo de las nuevas tecnologías aplicadas a la educación no se ha quedado ajeno al diseño y aplicación de las rúbricas en entornos virtuales, lo que conocemos como eRúbricas. Nuestro reto ha consistido en poner en diálogo un instrumento de seguimiento y evaluación virtual (eRúbricas) con tareas de aprendizaje presenciales en el aula que favorecen competencias específicas de módulo a la vez que transversales de grado. En dos de éstas últimas, Trabajo en equipo y Comunicación oral, son en las que hemos puesto nuestro punto de mira y sobre las cuales se ha articulado este trabajo.

En cuanto a la competencia de Trabajo en equipo la dimensión de Objetivos es en la que mejor vienen preparados los estudiantes, saben qué hay que hacer y lo tienen presente a lo largo de las tareas. Esto es reflejo, en definitiva, de la trayectoria escolar del alumnado en la que el producto y su consecución han primado frente al proceso de construcción del resultado. En cambio, en la dimensión de Participación, donde la intervención docente queda más diluida, por no tomar parte en la vida interna de los equipos de trabajo, es donde las valoraciones son más bajas, aunque experimentan un aumento considerable al aproximarse el final del cuatrimestre, donde de nuevo la presión por la entrega del producto se hace visible. Lo mismo ocurre con la dimensión relativa al Contenido perteneciente a la competencia de Comunicación oral. No es de extrañar que sean las dimensiones que la tradición escolar ha estado reforzando a lo largo de los años, en las que el alumnado se autoevalúa y evalúa a sus compañeros con valoraciones más altas (Objetivos y Contenidos). En cambio, son aquellas dimensiones como Participación (gestión y control de la toma de la palabra en el equipo de trabajo) y Recursos (soportes que apoyan el discurso) las que experimentan puntuaciones más bajas en las valoraciones debido a que aún estamos en un momento de tránsito desde el modelo que ha primado a lo largo de la historia en el que la enseñanza ha sido el eje del proceso educativo, al que se aboga en la actualidad donde el centro se ancla en la actividad del alumnado para el desarrollo de competencias (Perrenoud, 2004; Gimeno, 2008; Monereo, 2009; Blanco, 2009). 
El EEES resalta la importancia de centrar la enseñanza en los aprendizajes de los estudiantes, y que sean éstos quienes alcancen las competencias en un proceso de autoaprendizaje guiado por los docentes. Esta acción de guiar se materializa en el planteamiento metodológico de las tareas de aprendizaje (Zabala y Arnau, 2007). Las eRúbricas diseñadas de autoevaluación y coevaluación, consisten en la valoración del propio aprendizaje y el de sus compañeros, y de los factores que influyen en él. Un aspecto a destacar de estos instrumentos, es que son tareas de aprendizaje en si mismas, es decir: proporcionan al alumnado estrategias de desarrollo personal y profesional; ayudan a desarrollar la capacidad crítica; favorecen la autonomía; comprometen al alumnado en el proceso educativo; motivan para aprender; incrementan la responsabilidad con el aprendizaje; promueven la honestidad con los juicios y, proporcionan información al docente sobre su diseño y acción didáctica (Blanco, 2008; Buján, Rekalde y Aramendi, 2011; Serrano y Cebrián, 2011).

Las eRúbricas diseñadas para el desarrollo de las competencias transversales de Trabajo en equipo y Comunicación oral, permiten una evaluación formativa, cuya función ha sido potenciar la reflexión del alumnado sobre el avance o estancamiento en las dimensiones competenciales a lo largo del cuatrimestre y, por tanto, estas herramientas han servido para acompañar al alumnado, al facilitarles la valoración de su manera de hacer y su compromiso con las actividades, y analizar los cambios que le conviene incorporar para mejorar su desempeño (Gulikers et al., 2006). Es decir, han impulsado la activación de procesos de autorregulación y, desde ahí, estilos de aprendizaje más autónomos (Buján, Rekalde, y Aramendi, 2011).

El hecho de organizar el curriculum por módulos temáticos y compartir espacios entre las materias de tal manera que se planifiquen tareas interdisciplinares a lo largo del grado (Zabalza, 2012), le obliga al profesorado a consensuar y compartir criterios de evaluación (Rekalde, Martínez, y Marko, 2012) que pueden ser fácilmente socializados a través de las eRúbricas para el seguimiento y desarrollo de las competencias transversales, tanto en los espacios compartidos como en el espacio de la materia específica.

Las eRúbricas utilizadas como instrumentos formativos para la autoevaluación y coevaluación inciden en una dimensión ética, al establecer en su base la reflexión y la interrogación crítica como condiciones de mejora continua (Biggs, 1999). Esto se ha visto favorecido por la inmediatez del entorno virtual en el que se han ejecutado las valoraciones que ha permitido disponer de un feed-back inmediato (Serrano y Cebrián, 2011).

\section{A modo de conclusión}

Analizar las fortalezas y debilidades que puede presentar la utilización de eRúbricas en contextos de aprendizaje presenciales para el seguimiento y evaluación de las competencias transversales de trabajo en equipo y comunicación oral era el objetivo de este trabajo. De ahí que podamos concluir que:

Las eRúbricas para la evaluación de competencias transversales en la educación 
superior permiten compartir el diseño, la construcción, la utilización, la evaluación formativa y sumativa del alumnado a todo un equipo docente. La costosa tarea de consensuar criterios de evaluación para tareas y acciones comunes para un grupo de docentes, se facilita con el uso compartido de una eRúbrica. Así mismo, las eRúbricas facilitan la proyección de estos criterios tanto a las tareas de espacios compartidos como a aquellas desarrolladas en el espacio de la materia específica.

Las eRúbricas facilitan la formación del alumnado en competencias transversales al incidir sobre la importancia que desempeña la evaluación de cara a la mejora en tanto que: a) valoran lo que ya han conseguido y lo que les falta por adquirir, b) dotan de sentido global a los contenidos respecto a conocimientos, habilidades y actitudes, c) contrastan procesos y resultados, d) aportan evidencias a la luz de los criterios conocidos, e) disponen de feed-back inmediato, y f) convierten el uso del instrumento en una actividad habitual con sentido ético.

No obstante, debemos seguir mejorando tanto el diseño como la utilización de estas herramientas y para ello el alumnado ha sido quien más nos ha ayudado en identificar las debilidades con las mismas:

1) El alumnado es consciente que el propio diseño de las eRúbricas, condicionado por las estrategias de aprendizaje impulsadas a lo largo del cuatrimestre, hace que en algunos casos se pierda, en la globalidad de los equipos, el desarrollo de las competencias individuales de cada estudiante.

2) El enfoque desde el que se ha trabajado implica solicitar a los estudiantes procesos de reflexión y autoevaluación continuados en el tiempo sobre su aprendizaje, y por tanto requiere investigar en nuevas estrategias metodológicas que permitan un papel destacado del alumnado en el aprendizaje y, especialmente, un mayor compromiso en los procesos de evaluación.

Como se recoge en una de las voces del estudio: "Puedo afirmar que los momentos más dulces, buenos y que los recuerdo con más cariño siempre son en grupo y no sola, y eso mismo me ocurre cuando aprendo". Compartimos con esta alumna ese mismo sentimiento a la hora de haber participado en el proyecto de investigación con colegas de otras muchas universidades del estado, del cual se deriva este trabajo.

\section{Referencias bibliográficas}

BIGGS, J. (1999). Calidad del aprendizaje universitario. Madrid: Narcea.

BIGLIA, B. y BONET-MARTÍ, J. (2009). La construcción de narrativas como método de investigación psico-social. Prácticas de escritura compartida. Forum: Qualitative Social Research, 10 (1), 8-20.

BLANCO, A (2008). La rúbrica útil para la evaluación de competencias, en L. Prieto (coord.), La enseñanza universitaria centrada en el aprendizaje, 171-188. Barcelona: Octaedro. 
BLANCO, A. (2009). Desarrollo y evaluación de competencias en educación superior. Madrid: Narcea.

BOUD, D. (1995). Assessment and learning: contradictory or complimentary? en P. Knight (ed.), Assessment for Learning in Higher Education, 35-48. London: Kogan Page/SEDA.

BUJÁN, K.; REKALDE, I. y ARAMENDI, P. (2011) (Coord.). La evaluación de competencias en la educación superior. Las rúbricas como instrumento de evaluación. Sevilla: Editorial MAD.

DELORS, J. (1996) (Pres.). La educación encierra un tesoro. Informe a la UNESCO de la Comisión internacional sobre la educación para el siglo XXI. Madrid: Santillana/UNESCO.

DENZIN, N.K. (1978). The research act. Nueva York: McGraw Hill.

DOLL, W.E. (1993). A post-Modern perspective on curriculum. New York: Teachers College Press.

EURYDICE (2002). Las Competencias Clave. Un concepto en expansión dentro de la educación general obligatoria. Madrid: Eurydice y la Comisión Europea (Dirección General de Educación y Cultura).

GIMENO, J. (coord.) (2008). Educar por competencias ¿Qué hay de nuevo?. Madrid: Morata.

GOÑI, J.M. (2005). El espacio europeo de educación superior, un reto para la universidad. Barcelona: Octaedro.

GULIKERS, J.T.M.; BASTIAENS, Th.J. y KIRSCHNER, P.A. (2006). Authentic assessment, student and teacher perceptions: the practical value of the fivedimensional framework. Journal of Vocational Education and Training, 58 (3), 3337.

HARGREAVES, A. (2003). Enseñar en la sociedad del conocimiento. Barcelona: Octaedro.

IMBERNON, F. y Medina, J.L. (2006). Metodología participativa en el aula universitaria. La participación del alumnado, en M. Martínez y S. Carrasco, Propuestas para el cambio docente en la universidad, 91-121. Barcelona: Octaedro.

JANESICK, V. (1994). The dance Qualitative Research Design, en N.K. Denzin y Y.S. Lincoln (Eds.), Handbook of qualitative research, 209-219. London: Sage Publication.

JURADO, F. (2009). El enfoque sobre competencias: Una perspectiva crítica para la educación. Revista Complutense de Educación, 20 (2), 343-354.

KITSANTAS, A. y ZIMMERMAN, B.J. (2009). College Students' Homework and Academic Achievement: The Mediating Role of Self-Regulatory Beliefs. Metacognition and Learning, 4 (2), 97-110. 
LECOMPTE, M. (1995). Un matrimonio conveniente: diseño de investigación cualitativa y estándares para la evaluación de programas. Revista Electrónica de Investigación y Evaluación Educativa, 1 (1).

LOE. Ley Orgánica de Educación. Ley orgánica 2/2006, BOE nº 106.

MARTÍNEZ, M. y PAYÀ, M. (2007). La formación de la ciudadanía en el Espacio Europeo de Educación Superior, en J.L. García (ed.), Formar ciudadanos europeos, 19-98. Madrid: Academia Europea de Ciencias y Artes.

MONEREO, C. (coord.) (2009). PISA como excusa. Repensar la evaluación para cambiar la enseñanza. Barcelona: Graó.

MOYA, J. (2008). Las competencias básicas en el diseño y el desarrollo del currículo. Revista Curriculum, 21, 57-78.

PANADERO, E. y JONSSON, A. (2013). The use of scoring rubrics for formative assessment purposes revisited: A review. Educational Research Review, 9, 129-144.

PANADERO, E.; Alonso, J. y Huerta, J.A. (2012). Rubrics and self-assessment scripts effects on self-regulation, learning and self-efficacy in secondary education. Learning and Individual Differences, 22, 806-813.

PÉREZ GÓMEZ, A.I. (2008). ¿Competencias o pensamiento práctico? La construcción de los significados de representación y de acción, en J. Gimeno Sacristán (comp.), Educar por competencias ¿qué hay de nuevo?, 59-101. Madrid: Morata.

PERRENOUD, PH. (2004). Diez nuevas competencias para enseñar. Barcelona: Graó.

PRAHALAD, C.K y HAMEL, G. (1990). The core competence of the corporation. Harvard Business Review.

PUJADAS, J. J. (Ed.) (2004). Etnografía. Barcelona: Editorial UOC.

REKALDE, I. (2011). ¿Cómo afrontar el trabajo fin de grado? Un problema o una oportunidad para culminar con el desarrollo de las competencias. Revista Complutense de Educación, 22 ( 2), 179-193.

REKALDE, I. y PÉREZ-SOSTOA, V. (2011). Aprendiendo a evaluar el trabajo en equipo. La autoevaluación y coevaluación como estrategias formativas. I Congreso Internacional Virtual de formación del profesorado, 661-669. Murcia: Edit. Um.

REKALDE, I.; MARTÍNEZ, B. y MARKO, J.I. (2012b). Los proyectos interdisciplinares de módulo: Una experiencia innovadora en el Grado de Educación Social. Revista de Docencia Universitaria, 10 (3), 209-237.

REKALDE, I; ALONSO M ${ }^{\mathrm{a}}$ J.; CRUZ, E.; ELEJALDE, M ${ }^{\mathrm{a}} \mathrm{J}$.; ESQUISABEL, A.; IBAÑEZ, I.; JAUREGI, P.; JAUREGIZAR, J.; LOBATO, C.; MARTÍNEZ, I.; PALOMARES, T. y RUIZ de GAUNA, M ${ }^{a}$ P. (2012a). Formación de coordinadores para consolidar el cambio curricular en los grados de la UPV/EHU, en P. Membiela; N. Casado y M ${ }^{\mathrm{a}}$ I. Cebreiros (editores), El Espacio Europeo de 
Educación Superior y la educación por competencias, 203-208. Ourense: Educación editora.

ROEGIERS, X. (2007). Pedagogía de la integración. Costa Rica: CECC.

RYCHEN, D.S. y SALGANIK, L.H. (2004). Definir y seleccionar las competencias fundamentales para la vida. México: FCE.

SERRANO, J. y CEBRIÁN, M. (2011). Study of the impact on student learning using the eRubric tool and peer assessment, en A. Méndez-Vilas (Ed.), Education in a technological world: coomunicating current and emerging research and technological efforts, 421-427. Badajoz: Edit Formatex Research Center.

STEVENS, D. y LEVI, A. (2005). Introduction to Rubrics. Canada: Stylus Publishing.

UNESCO (1970). La alfabetización funcional. Cómo y por qué. París: Organización de las acciones unidas para la educación, la ciencia y la cultura.

ZABALA, A. y ARNAU, L. (2007). 11 ideas clave. Cómo aprender y enseñar competencias. Barcelona: Graó.

ZABALZA, M.A. (2012). Articulación y rediseño curricular: el eterno desafío institucional. Revista de Docencia Universitaria, 10 (3), 17-48.

\section{Referencias digitales}

FERNÁNDEZ, A. (14 julio 2008) Evaluación de los estudiantes por competencias [formato

pdf]http://webs.uvigo.es/victce/images/documentos/EEES/ForoANECA/presentacio n_amparo_fernandez.pdf (consultado 20 de noviembre de 2011).

OCDE. Proyecto DeSeCo (1999) Definition and selection of competencies: Theoretical and conceptual foundations [formato html] http://www.deseco.admin.ch/bfs/deseco/ en/index/02.html (consultado 3 de abril de 2012).

SALGANIK L.H., RYCHEN D.S., MOSER U. y KONSTANT J. W. (1999) Definition and Selection of Competencies. Projects on Competencies in the OECD Context. Analysis of Theoretical and Conceptual Foundations [format pdf] http://www.deseco.admin.ch/bfs/deseco/en/index/02.parsys.53466.downloadList.62 701.DownloadFile.tmp/1999.projectsoncompetenciesanalysis.pdf (consultado 5 de mayo de 2012).

TUNING PROYECT. (2000) Educational Structures in Europe [formato html] http://www.unideusto.org/tuningeu/home.html (consultado 15 de junio de 2012). 


\section{Correspondencia con los autores}

Itziar REKALDE RODRÍGUEZ

Universidad del País Vasco/Euskal Herriko Unibertsitatea

Facultad de Filosofía y Ciencias de la Educación

Departamento de Didáctica y Organización escolar

Av. Tolosa, n 70, Donostia-San Sebastián

Teléfono: 943015616

e-mail: itziar.rekalde@ehu.es

Karmele BUJÁN VIDALES

Universidad del País Vasco/Euskal Herriko Unibertsitatea

Escuela Universitaria del Profesorado de Donostia-San Sebastián

Departamento de Didáctica y Organización escolar

Plaza Oñati, n 3, Donostia-San Sebastián

Teléfono: 943017039

e-mail: karmele.bujan@ehu.es 